\section{Professionalisierung der Notfallpflege}

\section{Aktionsbündnis veröffentlicht Positionspapier}

» Das multiprofessionelle Aktionsbündnis Notfallpflege veröffentlichte jetzt ein Positionspapier. Damit will es eine hochwertige Notfallversorgung sichern und den Status der Notfallpflege in Deutschland stärken. Die Ziele sind klar: Anerkennung der Notaufnahmen als eigenständige Funktionsbereiche sowie die Etablierung einer Fachkraftquote für fachweitergebildete Notfallpflegende. „Die Notfallpflege ist ein immens dynamisches Berufsfeld mit hohem Anforderungsprofil, für das wir qualifizierte Pflegekräfte mit fachübergreifendem Wissen benötigen. Sie müssen dem er- höhten Fehlerrisiko gewachsen sein, das die Notaufnahme gegenüber anderen Bereichen der klinischen Versorgungsbereichen auszeichnet“, erläutert Prof. Felix Walcher, Unterstützer des Aktionsbündnisses und Präsidiumsmitglied der Deutschen Interdisziplinären Vereinigung für Intensiv- und Notfallmedizin (DIVI). Initiiert wurde das Bündnis von der Pflegeexpertin Katrin Wedler, Wissenschaftliche Mitarbeiterin der Klinik für Unfallchirurgie am Universitätsklinikum Magdeburg.

\section{www.divi.de}

\section{Bundesrat billigt DRK-Gesetz \\ Unbefristete Gestellung weiterhin möglich}

» Gute Nachrichten für den Verband der Schwesternschaften vom DRK: Die vom Bundestag am 1. Juni 2017 beschlossene Änderung des DRK-Gesetzes wurde vom Bundesrat gebilligt und am 24. Juli 2017 im Bundesgesetzblatt veröffentlicht. Damit wird die unbefristete Gestellung von Rotkreuzschwestern weiterhin möglich sein. Die im Arbeitnehmerüberlassungsgesetz vorgesehene Überlassungshöchstdauer von 18 Monaten wird nicht mehr gelten.

www.rotkreuzschwestern.de

\title{
Fördermittel zu wenig genutzt
}

\section{Mittel für Pflegestellen werden kaum abgerufen}

» Überall fehlen Pflegekräfte. Dennoch nutzen die Kliniken das Pflegestellen-Förderprogramm der Bundesregierung bisher nur zögerlich. Wie der Vorstand der Deutschen Stiftung Patientenschutz Eugen Brysch gegenüber der Deutschen Presseagentur (dpa) erklärte, hat bisher nicht einmal die Hälfte der mehr als 1.200 berechtigten Kliniken Gelder aus dem PflegestellenFörderprogramm abgeschöpft.
Von der für das 2016 bereitgestellten Summe seien laut Brysch nur 52 Mio. Euro abgerufen und nur 1.400 Stellen geschaffen worden. Auch angesichts des Fachkräftemangels sieht Brysch bereits jetzt das Ziel in Gefahr, in drei Jahren 6.300 Stellen zu schaffen. Er forderte die Krankenhäuser auf, den Pflegeberuf durch bessere Bezahlung, aber auch durch mehr Verantwortung für die Mitarbeiter attraktiver zu machen.
Im Rahmen des Krankenhausstrukturgesetzes hatte die Bundesregierung 2016 ein Förderprogramm für Neueinstellungen und die Aufstockung von Teilzeitstellen in der Pflege aufgelegt. Für 2016 wurden 110 Mio. Euro, für 2017220 Mio. Euro und ab 2018 dauerhaft 330 Mio. Euro zur Verfügung gestellt. Damit soll gezielt die pflegerische Versorgung am Bett dauerhaft gefördert werden.

\section{Pflege-Auszubildende testen QuartrBack}

\section{Projekt für Menschen mit nachlassendem Gedächtnis}

» Das Bundesministerium für Bildung und Forschung (BMBF) fördert das Projekt QuartrBack, das speziell für Menschen mit nachlassendem Gedächtnis oder demenziellen Einschränkungen entwickelt wurde. Ziel von QuartrBack ist, dass sich Menschen möglichst lange in ihrer gewohnten Umgebung aufhalten und selbstständig leben können. Dafür wird ein Mix aus intelligenter Technik, professionellen Dienstleistungen und einem Hilfsnetzwerk aus Angehörigen, Nachbarn und Ehrenamtlichen eingesetzt. Dieses Netzwerk steht Nutzern von QuartrBack unterwegs zur Verfügung. So können sie auch im Alter am gesell- schaftlichen Geschehen teilhaben, ganz im Sinne des Mottos von QuartrBack „bewegen - begegnen - bleiben“. Mit dem sogenannten Bürger-ProfiTechnik-Mix sind individuelle Angebote möglich, erklärt Dr. Susan Smeaton, wissenschaftliche Leiterin des Innovationszentrums bei der Evangelischen Heimstiftung (EHS) in Stuttgart. „Menschen möchten bis ins hohe Alter und auch bei Krankheit weiterhin Teil des Quartiers und der Gemeinschaft vor Ort bleiben, und zwar nicht irgendwie, sondern so, wie sie es ihr Leben lang gewohnt waren. Dazu steuert QuartrBack einen maßgeblichen Anteil bei." Am Projekt beteiligt sind neben der Evangelischen Heimstiftung als Konsortialführer weitere Partner aus Pflege, Wissenschaft und Praxis. Die ersten Pretests führten Auszubildende mehrerer Altenpflegeschulen durch. Das Ergebnis: Mit einem intelligenten Mix aus professionellen Dienstleistungen, moderner Technik und Unterstützung aus dem persönlichen Umfeld kann QuartrBack Menschen helfen. Das Modellprojekt geht im Oktober 2017 in die Feldtestphase über. Ein halbes Jahr lang werden an drei Standorten das Helfernetz und die Technik geprüft.

www.quartrback.de 